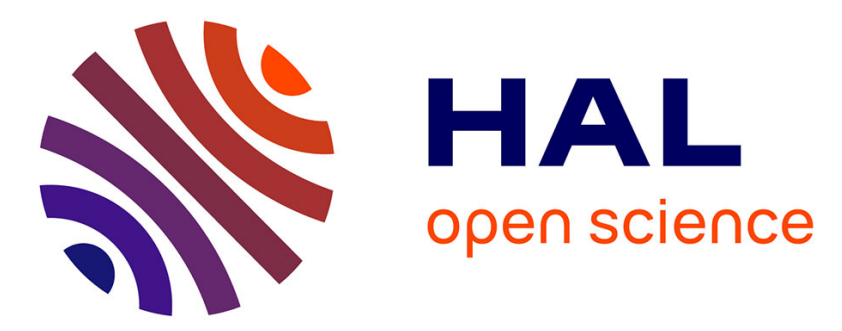

\title{
Motion Estimation-Based Image Enhancement in Ultrasound Imaging
}

\author{
Renaud Morin, Adrian Basarab, Stéphanie Bidon, Denis Kouamé
}

\section{To cite this version:}

Renaud Morin, Adrian Basarab, Stéphanie Bidon, Denis Kouamé. Motion Estimation-Based Image Enhancement in Ultrasound Imaging. Ultrasonics, 2015, 60, pp.19-26. 10.1016/j.ultras.2015.02.003 . hal-01278901

\section{HAL Id: hal-01278901 \\ https://hal.science/hal-01278901}

Submitted on 9 Mar 2016

HAL is a multi-disciplinary open access archive for the deposit and dissemination of scientific research documents, whether they are published or not. The documents may come from teaching and research institutions in France or abroad, or from public or private research centers.
L'archive ouverte pluridisciplinaire HAL, est destinée au dépôt et à la diffusion de documents scientifiques de niveau recherche, publiés ou non, émanant des établissements d'enseignement et de recherche français ou étrangers, des laboratoires publics ou privés. 


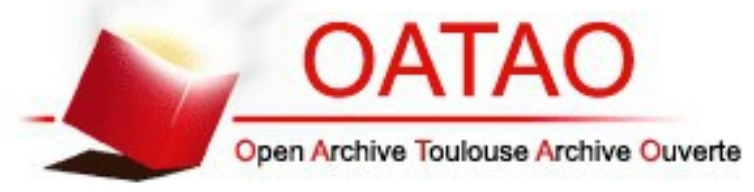

\section{Open Archive TOULOUSE Archive Ouverte (OATAO)}

OATAO is an open access repository that collects the work of Toulouse researchers and makes it freely available over the web where possible.

This is an author-deposited version published in : http://oatao.univ-toulouse.fr/ Eprints ID : 14818

To link to this article : DOI : $10.1016 / \mathrm{j}$. ultras.2015.02.003

URL : http://dx.doi.org/10.1016/j.ultras.2015.02.003

To cite this version : Morin, Renaud and Basarab, Adrian and Bidon, Stéphanie and Kouamé, Denis Motion Estimation-Based Image Enhancement in Ultrasound Imaging. (2015) Ultrasonics, vol. 60. pp. 19-26. ISSN 0041-624X

Any correspondance concerning this service should be sent to the repository administrator: staff-oatao@,listes-diff.inp-toulouse.fr 


\title{
Motion estimation-based image enhancement in ultrasound imaging
}

\author{
Renaud Morin ${ }^{\mathrm{a}, \mathrm{b}, *}$, Adrian Basarab $^{\mathrm{a}}$, Stéphanie Bidon ${ }^{\mathrm{c}}$, Denis Kouamé ${ }^{\mathrm{a}}$ \\ a University of Toulouse, IRIT - UMR CNRS 5505, Toulouse, France \\ ${ }^{\mathrm{b}}$ University of Dundee, Medical Research Institute, Ninewells Hospital \& Medical School, Scotland, United Kingdom \\ ${ }^{\mathrm{c}}$ University of Toulouse, ISAE, Department of Electronics Optronics and Signal, Toulouse, France
}

\begin{abstract}
A B S T R A C T
High resolution medical ultrasound (US) imaging is an ongoing challenge in many diagnosis applications and can be achieved either by instrumentation or by post-processing. Though many works have considered the issue of resolution enhancement in optical imaging, very few works have investigated this issue in US imaging. In optics, several algorithms have been proposed to achieve super-resolution (SR) image reconstruction, which consists of merging several low resolution images to create a higher resolution image. However, the straightforward implementation of such techniques for US imaging is unsuccessful, due to the interaction of ultrasound with tissue and speckle. We show how to overcome the limit of SR in this framework by refining the registration part of common multiframe techniques. For this purpose, we investigate motion estimation methods adapted to US imaging. Performance of the proposed technique is evaluated on both realistic simulated US images (providing an estimated best-case performance) and real US sequences of phantom and in-vivo thyroid images. Compared to classical SR methods, our technique brings both quantitative and qualitative improvements. Resolution gain was found to be 1.41 for the phantom sequence and 1.12 for the thyroid sequence and a quantitative study using the phantom further confirmed the spatial resolution enhancement. Furthermore, the contrast-to-noise ratio was increased by $27 \%$ and $13 \%$ for simulated and experimental US images, respectively.
\end{abstract}

\section{Introduction}

Among all the diagnostic imaging modalities, medical ultrasound (US) imaging is currently considered to be on the cutting edge of noninvasive technologies. Its cost-effectiveness, safety and portability are the main grounds of its common usage in the detection of various cancers, in the assessment of blood velocity or for the investigation of common biological soft tissues. Compared to other modalities such as magnetic resonance imaging or X-ray computed tomography, the various advantages of US imaging are however counterbalanced by its comparatively poor image quality. The main drawback of US images is their overall low contrast and their limited ability to distinguish between two small adjacent objects. Depending on the working frequency, which is related to the design of US transducers as well as the desired penetration depth, low spatial resolution can even further decrease the image quality $[1,2]$. Furthermore, due to underlying instrumentation constraints and random location of scatterers, US images are contaminated by an intrinsic noise called "speckle" which greatly reduces the general image quality and can hence lead to

\footnotetext{
* Corresponding author at: University of Dundee, Medical Research Institute,
} Ninewells Hospital \& Medical School, Scotland, United Kingdom. interpretation concerns. Nevertheless, in some cases, the speckle can be taken into account by a trained observer and may lead to complementary diagnostic information. The statistical properties of the speckle can, moreover, be exploited within several signal processing-based applications such as tissue characterisation and image segmentation [3].

As a result, over the past few decades, extensive efforts have been put into improving US image quality. In US, image quality can be improved in either pre- or post-processing. The former is usually achieved through the modernization of the US scanners, for instance by using high frequency transducers (at the cost of limited penetration depth [4]), backprojection image recovery methods [5] or by designing a proper adaptive beamforming $(\mathrm{ABF})$ algorithm such as Diffuse Time-domain Optimized Near-field Estimator (dTONE) [6] to replace the conventional delay and sum beamforming (at the cost of high computational load). Unfortunately, such techniques lead to tremendous instrumentation constraints that hinder the experimental reproducibility. An alternative consists of investigating post-processing resolution enhancement techniques similar to the works proposed in optical image or video fields $[7,8]$.

The so-called "super-resolution (SR) approach" was originally based on a sequence of low resolution (LR) optical images of the 


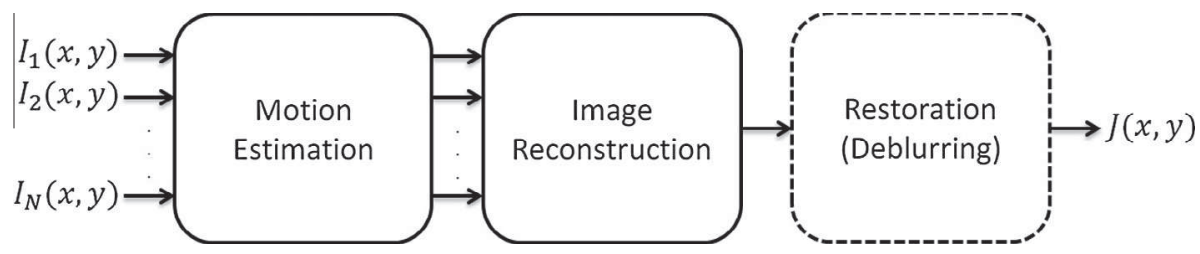

Fig. 1. The classical three-step scheme for super-resolution image reconstruction. $I_{1}(x, y), I_{2}(x, y), \ldots, I_{N}(x, y)$ are $N$ low resolution input images and $J(x, y)$ is the desired high resolution output image. The final restoration stage is optional and will not be addressed in this paper.

same scene using a frequency domain method [9]. The desired high resolution (HR) was obtained by using the relative sub-pixel motion between LR images and has been greatly improved [10] during the past decades. The straightforward implementation of such algorithms for US imaging was unsuccessful, due to the intrinsic nature of tissue elastic motions, speckle and the point spread function (PSF) [11]. Note that unlike strain compounding [12-14], which consists in averaging several ultrasound images acquired under different strain conditions in order to reduce the speckle, our goal is to improve the resolution of US images without altering their intrinsic characteristics.

In this paper, we show how to overcome the intrinsic limit of SR in the US imaging framework and how to preserve the native aspect of US images by refining the registration stage of common multiple frame SR before reconstructing the HR image. A realistic US simulation addressing the experimental limit of such a method is also provided.

\section{Motion estimation-based image enhancement in ultrasound imaging}

The main goal of this work is to address the SR reconstruction of US images when the motions can be estimated within sub-pixel accuracy. Here, the motion to be estimated for the HR image estimation is induced by the US transducer held by the practitioner and the associated compression of the local medium in the context of static elastography. This non-rigid tissue deformation has been used in many applications such as thyroid nodular disease characterisation [15]. More details regarding the magnitude of this motion can be found in Sections 4.1 and 4.2 for a sequence of phantom and thyroid images, respectively.

Note that any US image sequence exhibiting a non-integer displacement (in terms of number of samples) between consecutive frames can, in theory, be processed via the proposed method. This displacement may be natural (e.g. cardiac motion) or manually induced by the practitioner as in our case.

One should note that the overall displacement in the US sequences considered here is relatively small (especially in the thyroid case, where only a slight compression with the ultrasound probe has been applied) compared to the natural motion of the body (e.g., cardiac motion). In the case of image sequences with larger motions, the original low resolution images would be further decorrelated and would theoretically enhance the quality of the non-uniform interpolation process. This greater decorrelation would however be a drawback with respect to the registration step, whose accuracy is crucial in the proposed approach.

\subsection{Image sequence model}

Let $\mathbf{I}(x, y)=\left\{I_{1}(x, y), I_{2}(x, y), \ldots, I_{N}(x, y)\right\}$ be a given set of $N$ LR images. In order to create the desired HR image $J(x, y)$, the employed multiframe SR algorithm relies on a three-step scheme depicted in Fig. 1.
The first task deals with accurately estimating the motion between the $N$ input images. The relation between two consecutive frames is given by

$I_{n+1}(x, y)=I_{n}\left(x+u_{n}(x, y), y+v_{n}(x, y)\right)$

for $1 \leqslant n \leqslant N-1$, where $u_{n}(x, y)$ and $v_{n}(x, y)$ are the spatially varying displacement fields along the two directions between images $n$ and $n+1$. Various algorithms have been used to perform this motion estimation step, e.g., $[7,16]$ depending on the motion characteristics, but very few works have investigated applying it to SR in US [17].

Once both the estimated relative motions $\hat{\mathbf{u}}(x, y)=\left\{\hat{u}_{1}(x, y)\right.$, $\left.\hat{u}_{2}(x, y), \ldots, \hat{u}_{N-1}(x, y)\right\}$ and $\hat{\mathbf{v}}(x, y)=\left\{\hat{v}_{1}(x, y), \hat{v}_{2}(x, y), \ldots, \hat{v}_{N-1}(x, y)\right\}$ are computed, the $N$ images can be aligned according to a reference frame onto an HR grid, taking into account the motion estimation sub-pixel accuracy. The set of registered images is denoted by $\hat{\mathbf{I}}(x, y)=\left\{\hat{I}_{1}(x, y), \hat{I}_{2}(x, y), \ldots, \hat{I}_{N}(x, y)\right\}$. By convention, the first frame is considered as the reference frame, i.e., $\hat{I}_{1}(x, y)=I_{1}(x, y)$. Non-uniform interpolation is then performed onto this HR grid in order to fuse the $N$ images contained in $\hat{\mathbf{I}}(x, y)$. Unlike uniform interpolation, which would be employed on the set of images without pre-alignment, non-uniform interpolation processes the images registered using the motion estimated in the first step. In

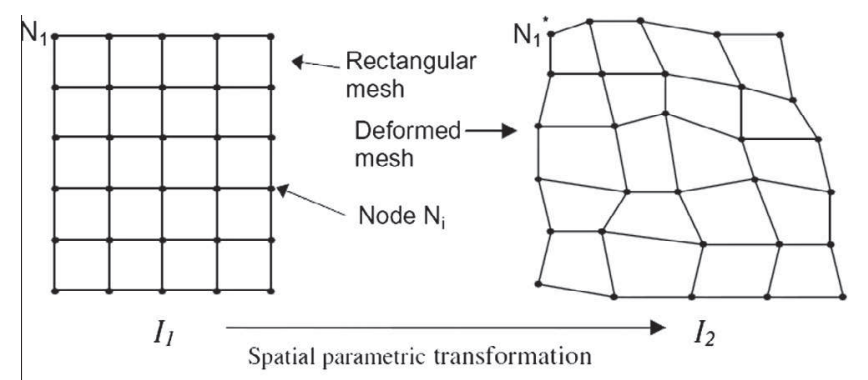

(a)

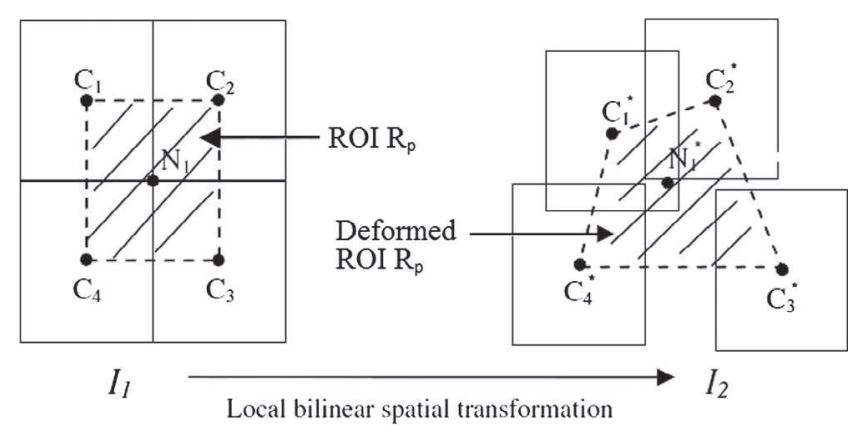

(b)

Fig. 2. Overview of BDBM, adapted from [15]. (a) Rectangular mesh on image $I_{1}$ and deformed mesh on image $I_{2}$. (b) Parametric estimation for a given region of interest (ROI) (hatched region) around one node. 


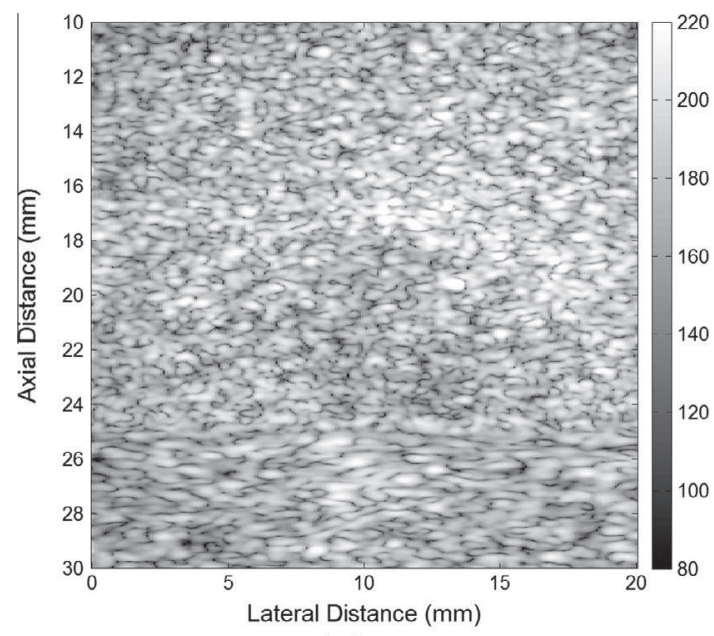

(a)

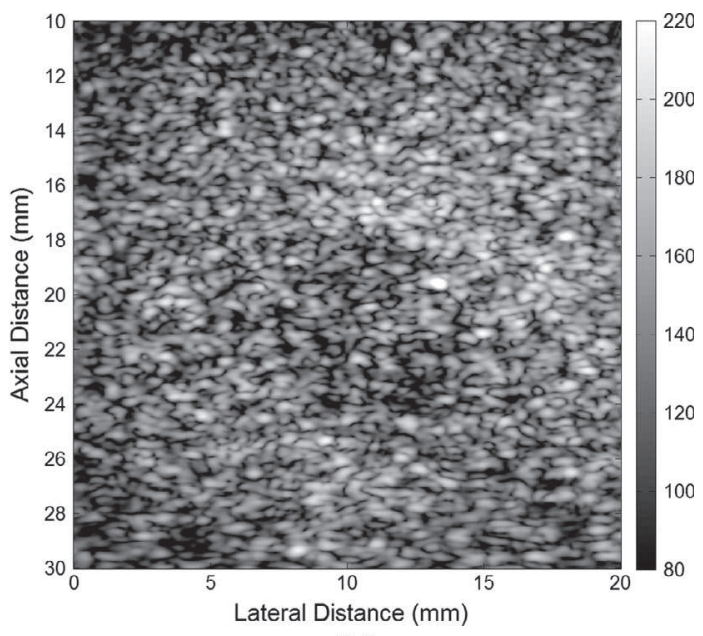

(b)

Fig. 3. Realistic simulation results of SR image reconstruction using a real US image as a template. (a) One of the 15 simulated LR input images. (b) Result of our method.

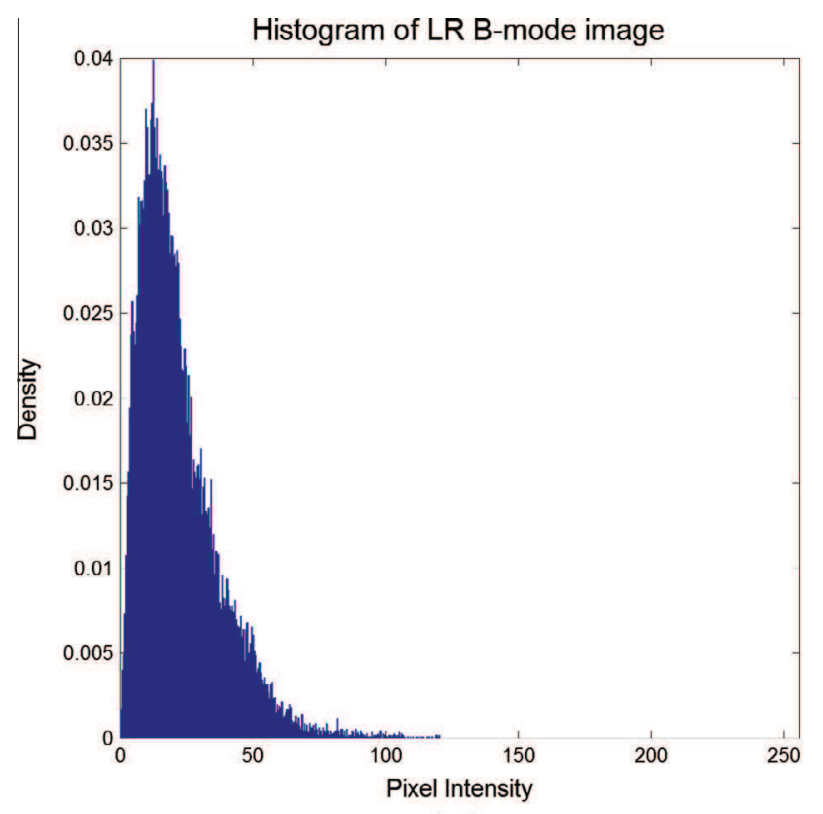

(a)

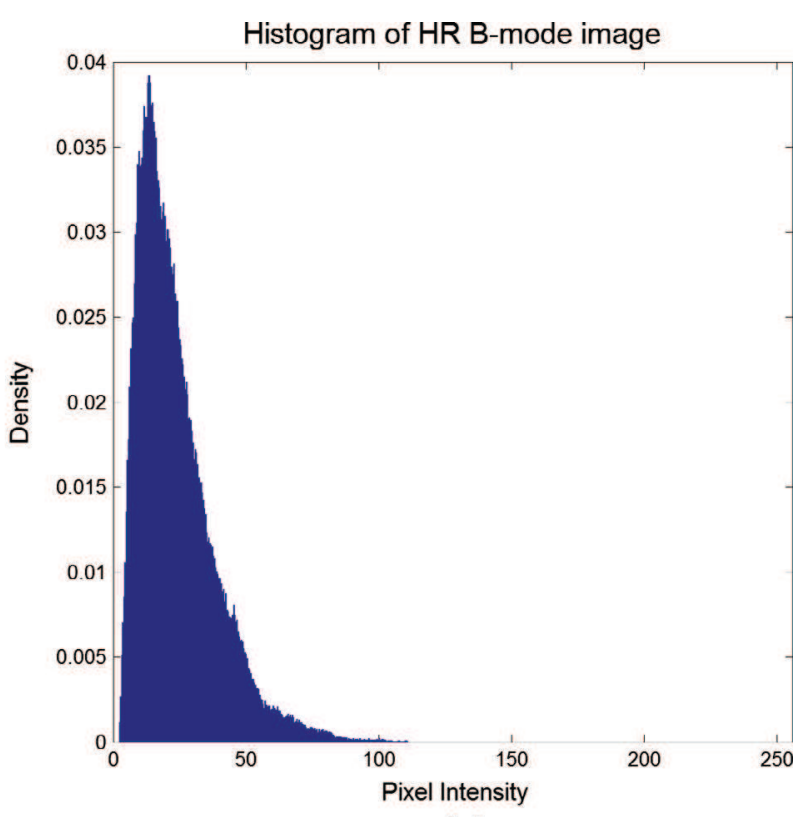

(b)

Fig. 4. Distribution of the (a) LR and (b) HR envelope data.

our work, we choose the bicubic interpolation as it achieves the best compromise between calculation time and quality of the resulting image [10].

A third deblurring step (not addressed in this paper, see [18,19]) can be added in order to enhance the output image $J(x, y)$, depending on the observation model and the available a priori information about the PSF.

Note that in the proposed framework, the motion estimation stage is the key element of the SR image reconstruction and will hence almost completely determine the quality of the resulting image. We have recently shown in [11] that classical motion estimation, usually involved in multiframe SR frameworks (see [10] and references therein), provide poor results when performed using US images. Indeed, these methods fail to estimate the true elastic motion and therefore break the speckle characteristics, resulting in an image degradation [11]. The main issue is that the motion estimation must be performed on the LR images with sub-pixel accuracy in order to provide improvements in the resulting HR image.

\subsection{Paper contribution}

Several methods have been proposed to perform the motion estimation task in US imaging, in various applications such as cardiology or elastography. Recent literature has shown the ongoing aspects of motion estimation in US imaging, with works such as [20]. A complete review of motion estimation algorithms in US imaging would however be out of the scope of this paper. Taking into account the nature of the tissue deformations exploited in this 


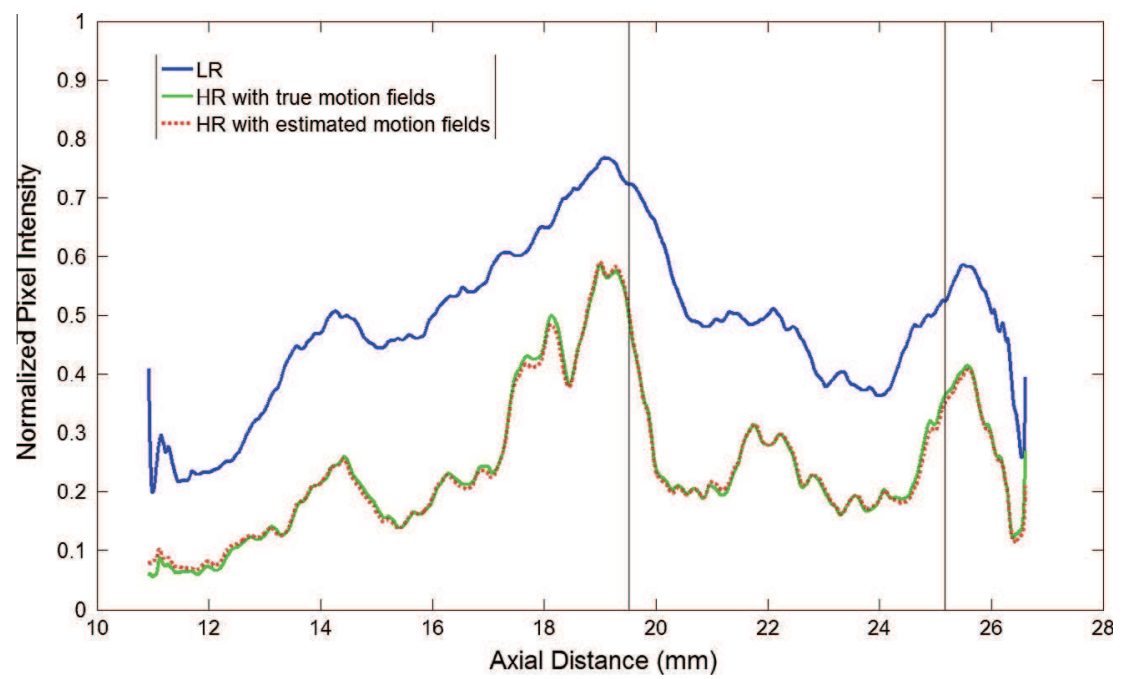

Fig. 5. Envelope of RF lines averaged around $10 \pm 1 \mathrm{~mm}$ in lateral distance for the phantom images in Fig. 3. The LR input image is in blue, the reconstructed HR output image using the true and the estimated displacement fields are in red and in green, respectively. Vertical straight lines provide the delimitation of the inclusion. (For interpretation of the references to color in this figure legend, the reader is referred to the web version of this article.)

paper (i.e., the elastographic fashion), we resorted to a recent motion estimation method designed for elastography.

This work relies entirely on a post-processing framework and is therefore not related to the constraints of the acquisition stage, such as the frame rate. SR image reconstruction is however compatible with a real time implementation using an efficient motion estimation algorithm and a sliding window update process commonly used in compound imaging [21], with no reduction in frame rate.

The motion estimation method used in this paper, referred to as Bilinear Deformable Block Matching (BDBM) [15,22], is adapted to complex tissue motion estimation and to small local deformations. Unlike classical block matching (BM), also called speckle tracking in US literature, BDBM is based on a local bilinear model and consequently takes into account the local deformations of the tissues. This method defines rectangular regions of interest (ROI) and a local parametrical motion model estimates the translations of its four corners, as shown in Fig. 2. Unlike the classical BM method that takes into account only rigid translations via two parameters $\left(d_{u}, d_{v}\right)$, this method uses a bilinear model with eight parameters to describe the local motion field:

$u(x, y)=a_{u} \cdot x+b_{u} \cdot y+c_{u} \cdot x \cdot y+d_{u}$

$v(x, y)=a_{v} \cdot x+b_{v} \cdot y+c_{v} \cdot x \cdot y+d_{v}$

where $u$ and $v$ are the displacements along lateral and axial directions and $a_{u, v}, b_{u, v}, c_{u, v}$ stand for the local scaling factors, rotations, and shears in each direction, respectively. The eight parameters of the bilinear transformation are locally estimated in the ROIs $R_{p}$ as shown in Fig. 2. For this purpose, in each $R_{p}$, BDBM resorts to classical BM in order to track each of the four corners.

The estimation of the bilinear parameters is refined using a multi-scale iterative approach, which consists of locally deforming the image with the estimated parameters of the previous iteration, and restarting the estimation by further refining the search regions (rectangular block of pixels defined around each corner in classical $\mathrm{BM})$. It has been shown that two iterations are sufficient in order to obtain good results [15]. For this reason, we also used two iterations $(K=2)$ in this paper. The main steps of the BDBM method for estimating the motion between two images are summarized in Algorithm 1 and its comprehensive description can be found in [15].
Algorithm 1. Motion estimation with BDBM [15,22]

Require: Unregistered input images $I_{n}(x, y)$ and $I_{n+1}(x, y)$.

Ensure: Registered output image $\hat{I}_{n+1}(x, y)$.

- Create initial rectangular mesh on $I_{n}$.

- Define regions of interest $R_{p}$ around nodes $N_{p}$.

for all $N_{p}$ do

- Initialize the translations of region of interest $R_{p}$ taking into account the estimation results of its neighbours [15].

for $k=1$ to $K$ do

- Consider four rectangular blocks denoted $B_{p q}$, around corners $C_{p q}$ of $R_{p}$, with $1 \leqslant q \leqslant 4$, and place four corresponding search regions on $I_{n+1}$.

- Interpolate the search regions by ratio $s_{u, k}$ in the lateral direction and $s_{v, k}$ in the axial direction.

- Estimate the translations of corners $C_{p q}$ of the current $R_{p}$ by simple block matching using the sum of absolute differences cost function four times.

- Compute the parameters of the bilinear model for the current study zone, which contains $R_{p}$ and $B_{p q}$.

- Deform the current study zone according to the previously computed parameters.

end for

end for

- Compute the dense motion field as detailed in [22].

- Compensate the motion to obtain $\hat{I}_{n+1}(x, y)$.

Once the motion for every image pair is estimated, the HR image can be reconstructed. All the LR images are first expressed in the coordinate frame of the reference image by accumulating the motion computed between successive frames of the sequence, as was performed for echocardiographic images in [23]. The image values are then interpolated on a regular HR grid using the sub-pixel motion information. As stated before, bicubic interpolation is chosen because of its low computational complexity and good results.

Several studies have investigated the optimal number of LR images to use when reconstructing the HR image [10]. This depends on many parameters, such as the registration accuracy, the imaging model or the total frequency content. Intuitively, 
one may think that the more LR images are used, the better the reconstruction should be. Nevertheless, there is a limit to the improvement that can be obtained: even from a very large number of LR images, it may not be possible to reconstruct a sharp HR image. Blur, noise, and inaccuracies in the signal model limit the increase in resolving power that can be obtained. The performance increases rapidly with the first images whereas the improvement is marginal beyond that (see, e.g., [10]).

Since this paper focuses on the registration part of the SR image reconstruction, the independent deconvolution problem (third step in Fig. 1) will not be addressed here but can be found in some related works $[18,19]$.

\section{Simulation of a realistic synthetic ultrasound sequence}

Performance of the SR image reconstruction scheme mainly depends on the accuracy of the motion estimation. In order to

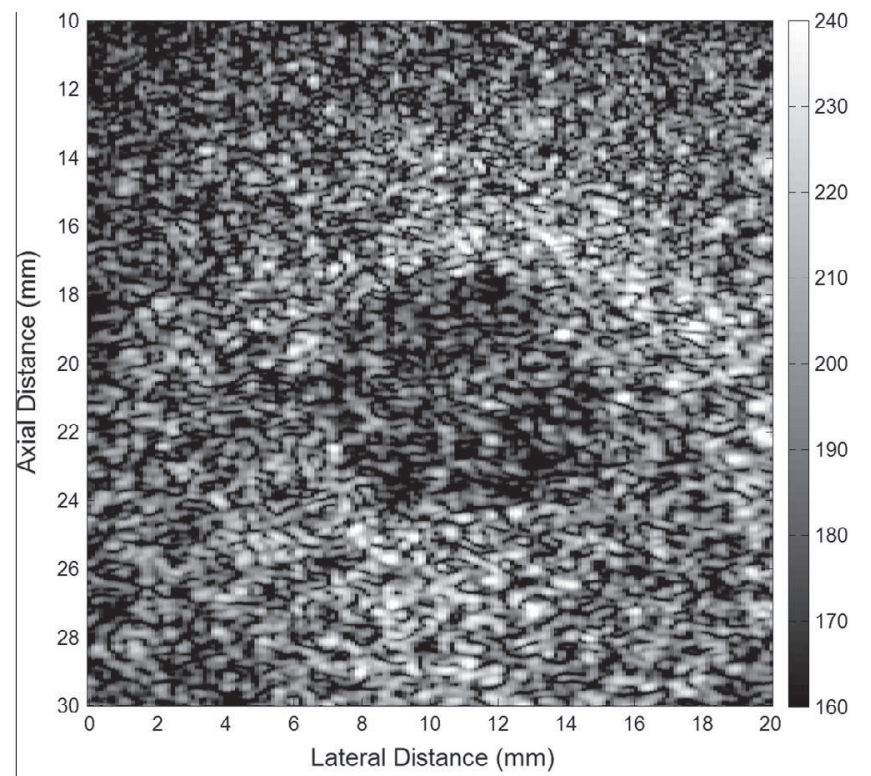

(a)

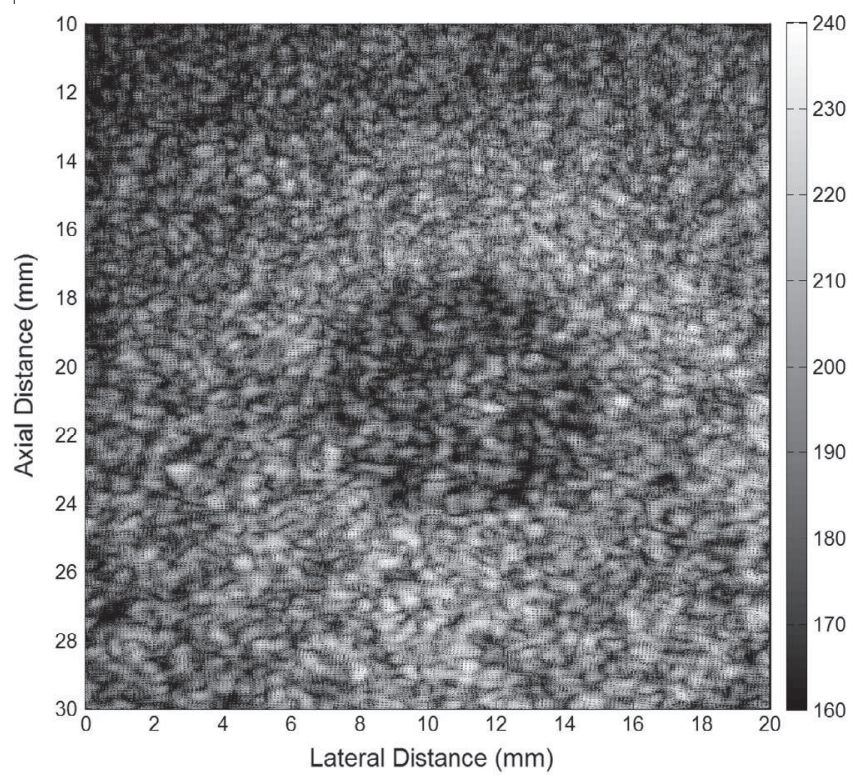

(c) assess both the feasibility and the limitations of our approach, we have used a framework for the generation of realistic simulated US sequences based on a true known motion [23]. The SR ratio related to the new spatial sampling of the HR image, which accounts for the pixel density increase in each direction, is set to 9 . Namely, a $50 \times 50$ pixel LR image would lead to a $450 \times 450$ pixel HR image.

The first frame of the synthetic sequence, $I_{s 1}(x, y)$, adopts an experimental US image as a template, denoted by $I_{1}(x, y)$. We used a phantom image, which consists of a 5 -mm-diameter cylindrical inclusion acquired with a $7.5 \mathrm{MHz}$ linear probe, as the template. The first step for the simulation of $I_{s 1}(x, y)$ deals with the generation of a scatter map with the set of scatter points positioned according to a uniform distribution [24]. The size of the simulated US image is $30.04 \mathrm{~mm} \times 34.72 \mathrm{~mm}$, or 2311 pixels $\times 217$ pixels axially and laterally, respectively. The number of scatterers $S$, set to $5 \times 10^{4}$ in our experiments, is chosen so that the speckle is fully

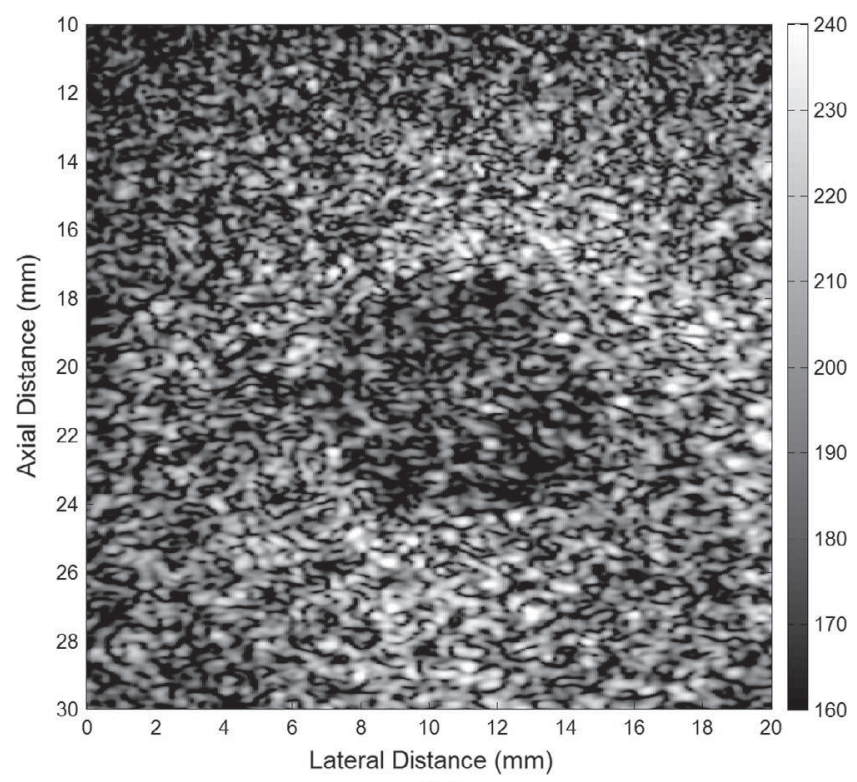

(b)

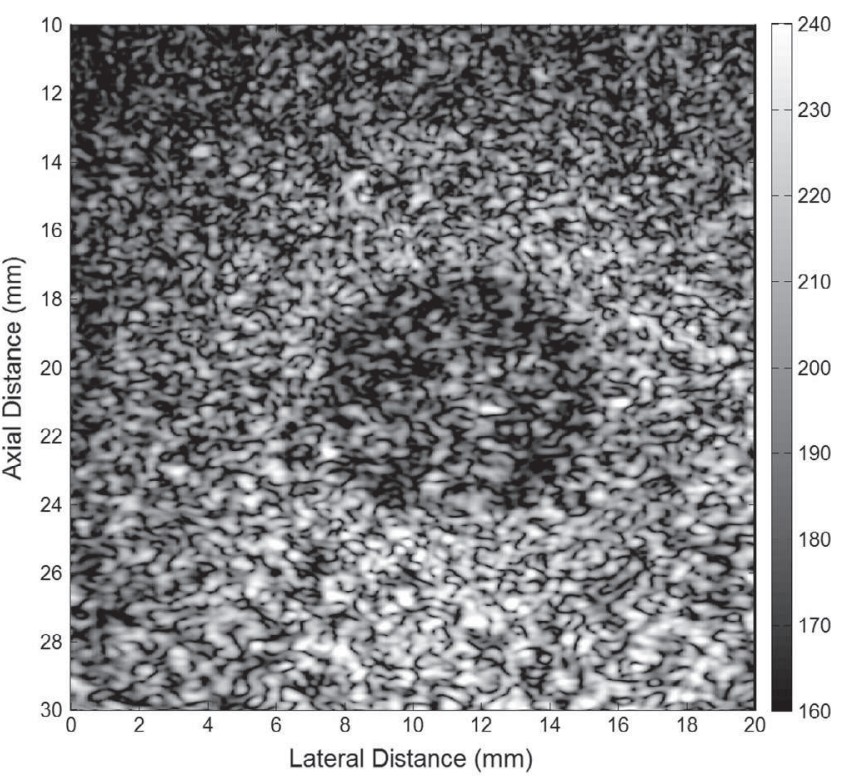

(d)

Fig. 6. Results of SR image reconstruction using various methods. (a) One of the 15 LR input images and the resulting HR output images using (b) a bicubic interpolation, (c) classic SR [10], and (d) our method. 
developed and the simulated image is visually acceptable. Position and amplitude of the $i$ th scatterer of frame $n$ are denoted by $\left(x_{i, n}, y_{i, n}\right)$ and $a_{i, n}$, respectively.

The scatterer amplitudes for the first frame are obtained by sampling the value of a template image $\tilde{I}_{1}(x, y)$ at the corresponding coordinates

$a_{i, 1}=\tilde{I}_{1}\left(x_{i, 1}, y_{i, 1}\right), \quad 1 \leqslant i \leqslant S$

where $\tilde{I}_{1}(x, y)$ is the (bicubic) interpolated envelope of the real US radio-frequency (RF) image $I_{1}(x, y)$, before the B-mode log-compression as stated in $[23,25]$.

The following frames of the sequence are then obtained by simply displacing the scatter map according to a known estimated motion, i.e., for $1 \leqslant i \leqslant S, 1 \leqslant n \leqslant N_{f}-1$,

$\left\{\begin{array}{l}x_{i, n+1}=x_{i, n}+\hat{u}_{n}(x, y) \\ y_{i, n+1}=y_{i, n}+\hat{v}_{n}(x, y)\end{array}\right.$

where $N_{f}$ stands for the total number of frames in the simulated sequence and $\hat{u}_{n}(x, y)$ and $\hat{v}_{n}(x, y)$ are the known motions in both directions. To ensure realism, the motion introduced in the simulated sequence is derived from a previous estimation performed on a real phantom image sequence, hence the term "realism". As this previous estimation may generate some errors and irregularities in the estimated motion fields, a slight low-pass filter is applied to these motion fields beforehand, as a pre-processing stage to the sequence simulation.

The amplitude of the displaced scatterers remains the same for all the frames of the sequence in order to preserve the speckle coherence. Namely, for $1 \leqslant i \leqslant S, 1 \leqslant n \leqslant N_{f}-1$,

$a_{i, n}=a_{i, 1}$

Once the complete sequence of scatter maps is computed, the associated US image is synthesized with Field II [24]. In the software parametrization, we used the linear transducer features with which the phantom was originally acquired: $7.5 \mathrm{MHz}$ center frequency, $60 \mathrm{MHz}$ sampling frequency, 512 physical elements of size $5 \mathrm{~mm} \times 0.2 \mathrm{~mm}$ (height and width, respectively), with 128 active elements.

Fig. 3 shows one frame of a realistic simulated US sequence and the reconstructed HR image using the estimated motion fields. The distributions of the simulated LR data and the reconstructed HR data are shown in Fig. 4. A fit to a Rayleigh distribution [3] was computed and the corresponding parameter $\sigma$ was estimated in both cases using the raylfit MATLAB function, resulting in $\sigma=19.03$ for the LR data and $\sigma=19.72$ for the HR data. This small $3.6 \%$ change in the distribution parameter confirms the preservation of the US image statistical properties. US B-mode profiles of $\mathrm{RF}$ lines passing through the cylindrical inclusion are shown in Fig. 5. The RF lines located around $10 \pm 1 \mathrm{~mm}$ in lateral distance were averaged using a moving average and presented together with the theoretical delineation of the phantom (two vertical lines).

It is important to notice that here, the SR image reconstruction is performed both with the true known motion used in the simulation of the synthetic US sequence and with motion fields estimated with the BDBM method on the synthetic sequence. The resulting HR images are visually consistent, as highlighted by the profiles in Fig. 5. Therefore, this realistic simulation gives the experimental limit of the method performance, showing that the chosen motion estimation algorithm is accurate enough for our application.

\section{Results}

\subsection{In-vitro phantom images}

The process described in Section 2 is assessed using US sequences containing 15 images of the $50 \times 50 \times 50 \mathrm{~mm}$ phantom with the same cylindrical inclusion of $5 \mathrm{~mm}$ in diameter scanned with a $7.5 \mathrm{MHz}$ linear probe. The average displacements between two consecutive frames are $0.205 \mathrm{~mm}$ and $0.058 \mathrm{~mm}$ in the axial and lateral directions, respectively. Fig. 6 shows one of the fifteen LR input images and the HR output image. As in Fig. 5, B-mode profiles of RF lines are examined using a moving average in Fig. 7, together with the theoretical delineation of the phantom (two vertical lines). Again, note that the horizontal (axial) distance in Fig. 6 corresponds to the vertical distance in Fig. 7. Furthermore, Figs. 6 and 7 can be seen as the experimental versions of the results presented in Figs. 3 and 5.

Qualitative improvements are observable as the inclusion is more accurately identified while both the structure of the speckle and the general aspect of the US image are preserved. The fine cylindrical structure of the inclusion is also recovered with the HR image.

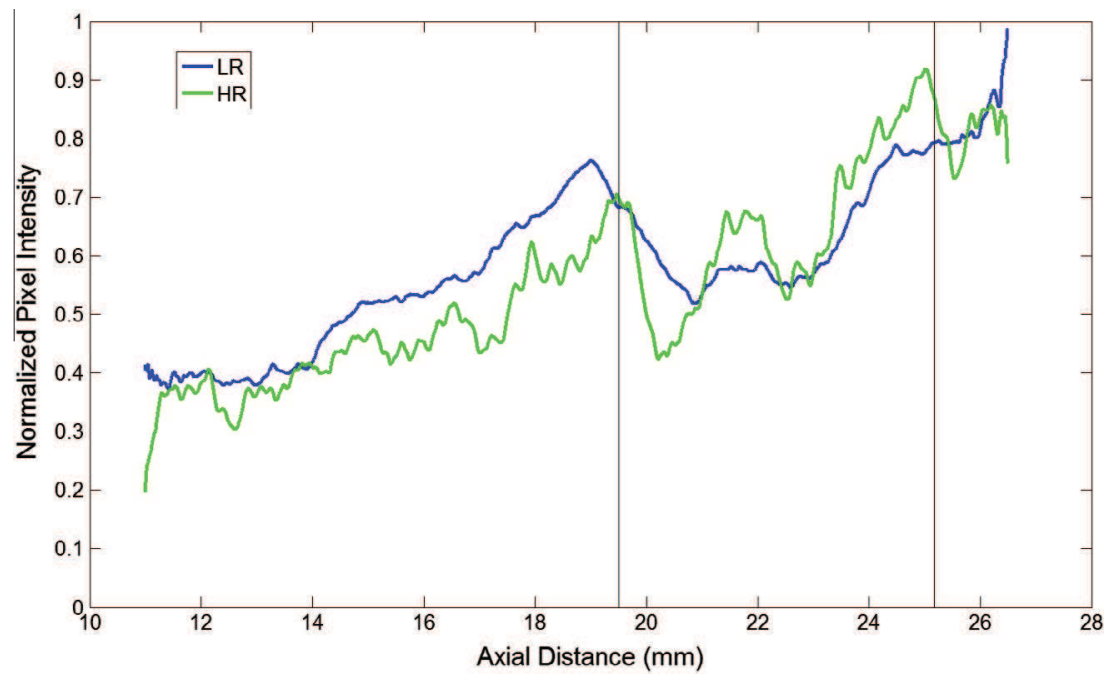

Fig. 7. Envelope of RF lines averaged around $10 \pm 1 \mathrm{~mm}$ in lateral distance for the phantom images in Fig. 6 . The LR input image is in blue and the reconstructed HR output image is in green. Vertical straight lines provide the delimitation of the inclusion. (For interpretation of the references to color in this figure legend, the reader is referred to the web version of this article.) 
A numerical measure of the resolution gain (RG) [26,27] was computed in order to assess the size of the speckle for LR and HR images. The RG criterion is defined by the ratio between the number of pixels of the normalized autocorrelation functions with values higher than 0.75 , computed for the LR envelope image and for the HR image. A square region of $15 \times 15 \mathrm{~mm}^{2}$ centred on the inclusion was used for each image and the gain was found to be 1.41. The improvement is modest and is related to the fact that the speckle spot size is not significantly modified in the reconstructed image. This gain could be further enhanced by using, for instance, deconvolution methods as a final step in the process described in Fig. 1.

Table 1

Width and slope of the inclusion interfaces in the simulated and the experimental phantom images. "Interface 1" and "Interface 2" (vertical straight lines in Fig. 5) correspond to the upper and lower regions, respectively, between the inclusion and the surrounding medium. Best results are shown in bold for each case.

\begin{tabular}{lllll}
\hline Images & Simulation & \multicolumn{2}{c}{ Experimentation } \\
& LR & HR & LR & HR \\
\hline Interface 1 width (mm) & 1.48 & $\mathbf{0 . 7 6}$ & 1.98 & $\mathbf{0 . 5 3}$ \\
Interface 2 width (mm) & 1.47 & $\mathbf{1 . 1 0}$ & $\mathbf{1 . 9 3}$ & 2.14 \\
Interface 1 mean slope & 3.08 & $\mathbf{7 . 2 7}$ & 2.46 & $\mathbf{9 . 9 1}$ \\
Interface 2 mean slope & 2.64 & $\mathbf{3 . 7 9}$ & 2.47 & $\mathbf{3 . 4 2}$
\end{tabular}

Table 2

Contrast-to-noise ratio comparison for the phantom images. Best results are shown in bold for each case.

\begin{tabular}{|c|c|c|c|c|}
\hline Images & LR & Interpolation & Classic & Our method \\
\hline \multicolumn{5}{|c|}{ Simulated phantom images } \\
\hline $\begin{array}{l}\mathrm{CNR}\left(\times 10^{-3}\right) \\
\text { (increase) }\end{array}$ & $\begin{array}{l}331.72 \\
(-)\end{array}$ & $\begin{array}{l}335.62 \\
(+1.5 \%)\end{array}$ & $\begin{array}{l}332.08 \\
(+0.3 \%)\end{array}$ & $\begin{array}{l}\mathbf{4 2 1 . 8 0} \\
(+27.2 \%)\end{array}$ \\
\hline \multicolumn{5}{|c|}{ Experimental phantom images } \\
\hline Images & LR & interpolation & classic & our method \\
\hline $\mathrm{CNR}\left(\times 10^{-3}\right)$ & 507.90 & 528.87 & 522.67 & 573.65 \\
\hline (increase) & $(-)$ & $(+4.1 \%)$ & $(+2.9 \%)$ & $(+12.9 \%)$ \\
\hline
\end{tabular}

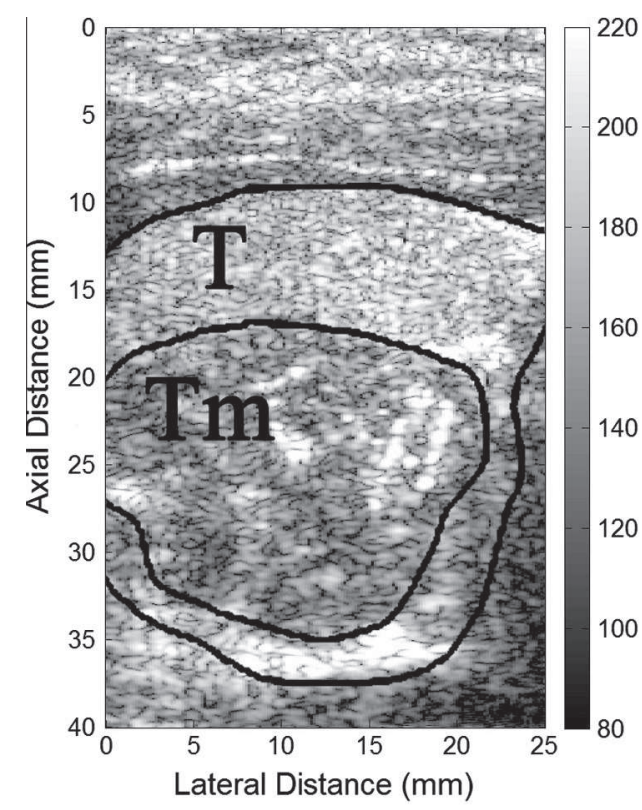

(a)
Two other quantitative measures were used in order to highlight the contribution of our approach in terms of contrast and structure. The contrast resolution enhancement was assessed in terms of contrast-to-noise ratio (CNR), a criterion often involved in the evaluation of US image processing techniques [22,28]. The CNR is defined as

$\mathrm{CNR}=\frac{\left|\mu_{i}-\mu_{o}\right|}{\sqrt{\sigma_{i}^{2}+\sigma_{o}^{2}}}$

where $\mu_{i}$ and $\mu_{o}$ are the means of regions inside and outside the inclusion, respectively, and $\sigma_{i}^{2}$ and $\sigma_{o}^{2}$ are the corresponding variances. The outer regions were chosen at the same depth as the inner region for a legitimate comparison. The CNR was computed on $4 \mathrm{~mm} \times 4 \mathrm{~mm}$ regions of approximately $15 \times 10^{4}$ samples and was increased by approximately $13 \%$ compared to the original LR image, as summarized in Table 2, for the simulated and the in vitro phantoms. The CNR was successively computed using one LR input image and the three HR images based on bicubic interpolation, classic SR as reported in [11] and our method (see the corresponding images in Fig. 6). The greater improvement is achieved in the phantom simulation since the ground truth motion is perfectly known.

It is important to note that the improvement of any given quantitative measure is only an indication and cannot reflect the algorithm's general behaviour. Thus, though the classic HR image leads to an increase of $2.9 \%$ in the CNR in Table 2, the general aspect of the image makes it impossible to exploit such a result. Note that the interpolation-based HR image improves the CNR whereas no additional information is provided in the reconstruction process.

The image structure enhancement was finally quantified by taking advantage of the characteristics of the phantom. The inclusion interface must be accurately delineated and the transition between the inner and the outer regions should be as narrow as possible in the B-mode images. The related gain can thus be thought of as the improvement in the transition width and slope, as summarized in Table 1 . The interface width was estimated as the region located

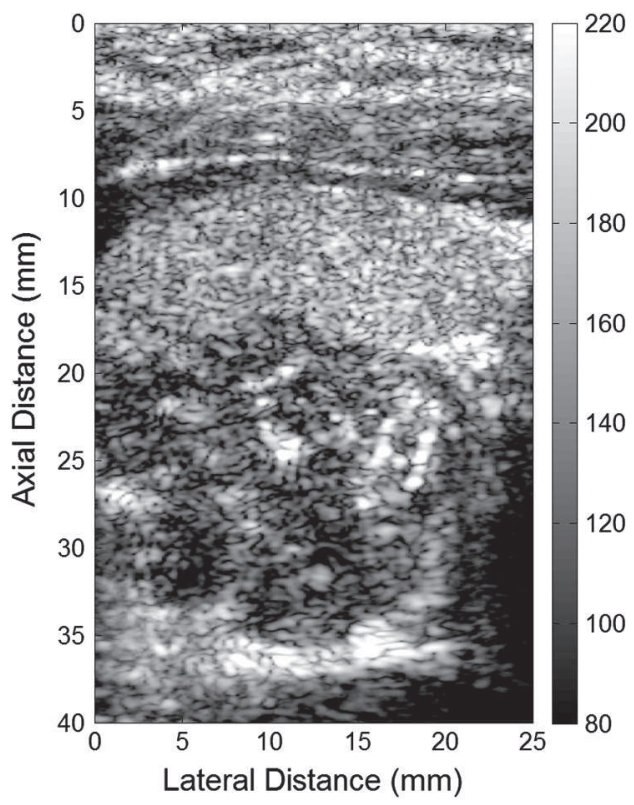

(b)

Fig. 8. Experimental results of SR image reconstruction on in vivo thyroid images with a malignant tumour delineated by a specialist doctor. T - thyroid gland, Tm malignant tumour. (a) One of the 15 LR input images. (b) Resulting HR output image. 
around the inclusion delineations, between the two main surrounding peaks. Its slope was computed using a linear least squares fitting. Note that these peaks may not always clearly exist and are dependent on the LR image native quality. Table 1 shows that the HR reconstructed images of the phantom have a better delineation of the inclusion interfaces in almost every case.

\subsection{In-vivo thyroid images}

The proposed algorithm was also used to process 15 US frames of an in vivo thyroid with a malignant tumour. The images were initially acquired for US static elastography, while applying a small compression with the US probe. The average displacements between two consecutive frames are $0.031 \mathrm{~mm}$ and $0.046 \mathrm{~mm}$ in the axial and lateral directions, respectively. More details about the thyroid data may be found in [22]. The results are displayed in Fig. 8. Though the evaluation of in vivo data is more complicated, results show a better accuracy of the thyroid overall structure according to the delineation provided by a specialist doctor and shown in Fig. 8(a). Though confirmed by elastograms in [23], this manual delineation might not be entirely accurate and is hence used here for indication only.

The RG was computed using a square region of $15 \times 15 \mathrm{~mm}^{2}$ located on the boundary between healthy and tumoral regions, resulting in a moderate gain of 1.12 .

The CNR was calculated in a slightly different way than in Section 4.1 since the inner and outer regions are not clearly defined. The inner region was chosen in the tumour region (dark lower part of the thyroid) while the outer region was rather chosen in the healthy region (bright upper part of the thyroid). The delineations of these regions were hand-drawn by a specialist doctor. The CNR was thus increased by approximately $24 \%$ compared to the original LR thyroid image.

\section{Conclusion}

This work proposed a framework for the image enhancement of ultrasound images based on a set of low-resolution ultrasound frames. The key steps of this reconstruction scheme are motion compensation and image reconstruction. Elastic motion estimation and bicubic interpolation are performed in order to better reconstruct the high-resolution output image, enhancing the overall image quality and preserving the ultrasound image nature. The algorithm performance is assessed using synthetic and in vivo ultrasound data and shows improvements in both qualitative and quantitative ways. In vivo ultrasound thyroid images are eventually processed and enhanced in the same way, thus demonstrating the various capabilities of this promising reconstruction technique in ultrasound imaging.

Future works will involve the compensation of the registration errors during a more robust interpolation stage by further taking into account the statistics of ultrasound images. To confirm the results presented in this paper, further investigations need to be conducted using a more extensive dataset. It would also be desirable to investigate the use of super-resolution image reconstruction in combination with deconvolution approaches via the incorporation of information related to the point spread function.

\section{Acknowledgment} grant.

\section{References}

[1] T.L. Szabo, Diagnostic Ultrasound Imaging: Inside Out, Biomedical Engineering Series, Elsevier, Academic Press, 2004

[2] J.L. Prince, J.M. Links, Medical imaging signals and systems, Pearson Prentice Hall, 2006.

[3] M. Pereyra, N. Dobigeon, H. Batatia, J.-Y. Tourneret, Segmentation of skin lesions in 2-D and 3-D ultrasound images using a spatially coherent generalized Rayleigh mixture model, IEEE Trans. Med. Imag. 31 (2012) 1509-1520.

[4] F. Foster, C.J. Pavlin, K.A. Harasiewicz, D.A. Christopher, D.H. Turnbull, Advances in ultrasound biomicroscopy, Ultrasound Med. Biol. 26 (2000) 1-27.

[5] G.T. Clement, J. Huttunen, K. Hynynen, Superresolution ultrasound imaging using back-projected reconstruction, J. Acoust. Soc. Am. 118 (2005) 39533960 .

[6] M.A. Ellis, F. Viola, W.F. Walker, Super-resolution image reconstruction using diffuse source models, Ultrasound Med. Biol. 36 (2010) 967-977.

[7] S.C. Park, M.K. Park, M.G. Kang, Super-resolution image reconstruction: a technical overview, IEEE Signal Process. Mag. 20 (2003) 21-36.

[8] P. Milanfar, Super-Resolution Imaging, Digital Imaging and Computer Vision, Taylor \& Francis Group, 2010.

[9] R.Y. Tsai, T.S. Huang, Multiframe image restoration and registration, in: C.T. Greenwich (Ed.), Advances in Computer Vision and Image Processing, vol. 1, JAI Press, 1984, pp. 317-339.

[10] P. Vandewalle, S. Süsstrunk, M. Vetterli, A frequency domain approach to registration of aliased images with application to super-resolution, EURASIP J. Appl. Signal Process. 2006 (2006) 1-14.

[11] R. Morin, A. Basarab, M. Ploquin, D. Kouamé, Post-processing multiple-frame super-resolution in ultrasound imaging, in: Proc. SPIE Medical Imaging, vol. 8320, 2012, 83201G-83201G-8, http://dx.doi.org/10.1117/12.910711.

[12] P.-C. Li, C.-L. Wu, Strain compounding: spatial resolution and performance on human images, Ultrasound Med. Biol. 27 (2001) 1535-1541.

[13] P.-C. Li, M.-J. Chen, Strain compounding: a new approach for speckle reduction, IEEE Trans. Ultrason. Ferroelectr. Freq. Control 49 (2002) 39-46.

[14] C.-H. Lin, C.-M. Weng, Y.-N. Sun, Ultrasound image compounding based on motion compensation, in: IEEE-EMBS 2005, 27th Annual International Conference of the Engineering in Medicine and Biology Society, 2005, pp. 6445-6448, http://dx.doi.org/10.1109/IEMBS.2005.1615974.

[15] A. Basarab, H. Liebgott, F. Morestin, A. Lyshchik, T. Higashi, R. Asato, P. Delachartre, A method for vector displacement estimation with ultrasound imaging and its application for thyroid nodular disease, Med. Image Anal. 12 (2008) 259-274.

[16] B. Zitov, J. Flusser, Image registration methods: a survey, Image Vis. Comput. 21 (2003) 977-1000.

[17] H. Zhang, M. Wan, J. Wan, X. Qin, Super-resolution reconstruction of deformable tissue from temporal sequence of ultrasound images, in: International Conference on Artificial Intelligence and Computational Intelligence (AICI), vol. 1, 2010, pp. 337-342, http://dx.doi.org/10.1109/AICI. 2010.78 .

[18] R. Morin, A. Basarab, D. Kouamé, Alternating direction method of multipliers framework for super-resolution in ultrasound imaging, in: Proc. 2012 9th IEEE International Symposium on Biomedical Imaging (ISBI), 2012, pp. 1595-1598, http://dx.doi.org/10.1109/ISBI.2012.6235880.

[19] R. Morin, S. Bidon, A. Basarab, D. Kouamé, Semi-blind deconvolution for resolution enhancement in ultrasound imaging, in: Proc. 2013 20th IEEE International Conference on Image Processing (ICIP), 2013, pp. 1413-1417, http://dx.doi.org/10.1109/ICIP.2013.6738290.

[20] B. Byram, G.E. Trahey, M. Palmeri, Bayesian speckle tracking. Part I: An implementable perturbation to the likelihood function for ultrasound displacement estimation, IEEE Trans. Ultrason. Ferroelectr. Freq. Control 60 (2013) 132-143.

[21] R.R. Entrekin, B.A. Porter, H.H. Sillesen, A.D. Wong, P.L. Cooperberg, C.H. Fix, Real-time spatial compound imaging: application to breast, vascular, and musculoskeletal ultrasound, Semin. Ultrasound CT MRI 22 (2001) 50-64.

[22] A. Basarab, A. Lyshchik, C. Grava, V. Buzuloiu, P. Delachartre, Ultrasound image sequence registration and its application for thyroid nodular disease, J. Signal Process. Syst. 55 (2009) 127-137.

[23] M. Alessandrini, H. Liebgott, D. Friboulet, O. Bernard, Simulation of realistic echocardiographic sequences for ground-truth validation of motion estimation, in: Proc. IEEE Int. Conf. Image Process. (ICIP), pp. 2329-2332.

[24] J.A. Jensen, Field: a program for simulating ultrasound systems, in: 10th Nordic-Baltic Conference on Biomedical Imaging, pp. 351-353.

[25] M. Alessandrini, A. Basarab, L. Boussel, X. Guo, A. Serusclat, D. Friboulet, D. Kouame, O. Bernard, H. Liebgott, A new technique for the estimation of cardiac motion in echocardiography based on transverse oscillations: a preliminary evaluation in silico and a feasibility demonstration in vivo, IEEE Trans. Med. Imag. 33 (2014) 1148-1162.

[26] J.A. Jensen, J. Mathorne, T. Gravesen, B. Stage, Deconvolution of in-vivo ultrasound B-mode images, Ultrason. Imag. 15 (1993) 122-133.

[27] O. Michailovich, A. Tannenbaum, Blind deconvolution of medical ultrasound images: a parametric inverse filtering approach, IEEE Trans. Image Process. 16 (2007) 3005-3019.

[28] E. Filoux, J. Mamou, O. Aristizabal, J.A. Ketterling, Estimation of spatial resolution for high-frequency imaging systems using a novel anechoic-sphere phantom, J. Acoust. Soc. Am. 128 (2010) 2280. 